\title{
Do Curriculum-Based Social and Emotional Learning Programs in Early Childhood Education and Care Strengthen Teacher Outcomes? A Systematic Literature Review
}

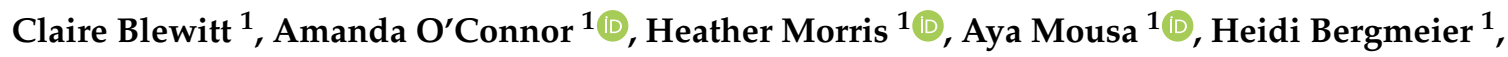 \\ Andrea Nolan ${ }^{2}{ }^{\oplus}$, Kylie Jackson ${ }^{3}$, Helen Barrett ${ }^{3}$ and Helen Skouteris ${ }^{1,4, *}$ \\ 1 Monash Centre for Health Research and Implementation, Monash University, \\ Melbourne, VIC 3168, Australia; claire.blewitt@monash.edu (C.B.); mandy.oconnor@monash.edu (A.O.); \\ heather.morris@monash.edu (H.M.); aya.mousa@monash.edu (A.M.); heidi.bergmeier@monash.edu (H.B.) \\ 2 School of Education, Faculty of Arts and Education, Deakin University, Geelong, VIC 3220, Australia; \\ a.nolan@deakin.edu.au \\ 3 Bestchance Child Family Care, Melbourne, VIC 3150, Australia; kjackson@bestchance.org.au (K.J.); \\ hbarrett@bestchance.org.au (H.B.) \\ 4 Warwick Business School, Warwick University, Coventry CV4 7AL, UK \\ * Correspondence: helen.skouteris@monash.edu
}

Received: 2 December 2019; Accepted: 1 February 2020; Published: 7 February 2020

\begin{abstract}
There is growing awareness of the benefits of curriculum-based social and emotional learning (SEL) programs in Early Childhood Education and Care settings for children's social, emotional, and cognitive development. While many SEL programs aim to strengthen teachers' capacity and capability to foster children's social and emotional skills, research effort has focused on understanding the impact on child outcomes, with less emphasis on improvement in teaching quality. This systematic literature review examined the effectiveness of universal curriculum-based SEL programs on teacher outcomes. Fifteen studies met inclusion criteria, capturing ten distinct SEL interventions. The findings suggest SEL programs may strengthen teaching quality, particularly the provision of responsive and nurturing teacher-child interactions and effective classroom management. Data were insufficient to ascertain whether participation improved teachers' knowledge, self-efficacy, or social-emotional wellbeing. The potential pathways between SEL intervention, teaching quality and children's developmental outcomes are discussed.
\end{abstract}

Keywords: preschool; kindergarten; social and emotional learning; social and emotional development; teaching quality; teacher-child interaction; pedagogy

\section{Introduction}

Engagement in Early Childhood Education and Care (ECEC) services can strengthen children's social-emotional and cognitive development, with benefits that persist over time [1]. The quality of these Early Learning programs is an important predictor of language and literacy skill, social-emotional competence, and behavioural engagement [2-5], particularly for children experiencing economic disadvantage [6-8].

\subsection{Importance of High-Quality ECEC for Children's Social-Emotional Development}

The quality of ECEC programs is influenced by the social, emotional, and instructional aspects of children's interactions with educators and peers (known as process quality) [9]. Process quality 
is influenced by the physical classroom environment, teacher-child ratio, group size, staff training and qualifications (known as structural quality), as well as teachers' own personal attributes. For example, high levels of self-efficacy have been associated with positive expectations for children [10], empathy [11], increased use of high-quality practices in preschool rooms [12], and time spent teaching social, emotional, and cognitive skills [13]. Educators' own social and emotional wellbeing can influence their ability to build strong relationships and facilitate positive outcomes for children [14], and teacher stress has predicted lower levels and less consistent emotional support [15] and lower quality teaching practices [13]. Empirical research and theory emphasize that high-quality teacher-child interactions are especially vital to children acquiring the social-emotional skills necessary to form prosocial relationships and engage in learning [3-5,16]. However, studies indicate many children are not consistently exposed to the quality of interactions required for optimal development [17-22].

\subsection{Social and Emotional Learning Programs in Early Learning Settings}

A growing number of social and emotional learning (SEL) programs designed for Early Learning settings focus on both high-quality teacher-child interactions and targeted pedagogy to nurture children's social and emotional development. The Collaborative for Academic, Social and Emotional Learning (CASEL) define SEL as the process through which children recognise, understand and regulate their emotions, empathize with the feelings and experiences of others, build and maintain prosocial relationships, establish and achieve positive goals, and make responsible decisions [23]. CASEL propose five competencies, comprising knowledge, skills, and attitude that underpin SEL: self-awareness, social awareness, self-management, relationship skills, and responsible decision-making. (Table 1). The application of SEL approaches in ECEC settings, however, requires careful consideration of the unique developmental characteristics of preschool-aged children, such as the emerging cognitive abilities that underpin social and self-awareness, and the limited ability to regulate behaviour compared to older children [24].

Table 1. Collaborative for Academic, Social and Emotional Learning (CASEL) Social and Emotional Learning Competencies.

\begin{tabular}{ll}
\hline \multicolumn{1}{c}{ CASEL Competence Domain } & \multicolumn{1}{c}{ Description [23] } \\
\hline Self-Awareness & $\begin{array}{l}\text { Recognising emotions, thoughts, strengths and limitations, self-confidence, } \\
\text { self-efficacy, understanding of how thoughts, feelings, and actions are connected. }\end{array}$ \\
\hline Social Awareness & Understanding and empathizing with others. \\
\hline Self-Management & $\begin{array}{l}\text { Effectively regulating emotions and behaviours, including impulse control and } \\
\text { perseverance. }\end{array}$ \\
\hline Relationship Skills & $\begin{array}{l}\text { Forming and maintaining prosocial relationships, communication, listening, } \\
\text { cooperation, managing conflict. }\end{array}$ \\
\hline Responsible Decision-Making & $\begin{array}{l}\text { Identifying and effectively solving social and behavioural problems, evaluating } \\
\text { consequences of actions. }\end{array}$ \\
\hline
\end{tabular}

Early childhood educators can foster children's SEL through a variety of approaches including explicit social-emotional skill instruction, child-centered practices and positive classroom management strategies that promote cooperation and prosocial behaviour, and integration within a wider pedagogy $[23,25]$. Programs that emphasize improving the quality of educator-child interactions, modifying the room environment or processes, or introducing different ways to structure peer interactions have an implicit focus on SEL. In contrast, explicit programs typically include a curriculum-based component, targeting social and emotional skills through instructional practices, modelling, and opportunities for practice across different contexts [23]. SEL programs may be delivered to all children within a group (universal programming) or be offered as an early intervention or treatment approach for children at risk of or experiencing social, emotional, or behavioural difficulties [23]. 


\subsection{Recent Research Reviews}

Most reviews examining the effectiveness of ECEC-based SEL intervention have focused on child outcomes, suggesting a small-to-moderate impact on children's social-emotional functioning [26-28]. A recent systematic review and meta-analysis by our research group of 79 controlled intervention studies (capturing 51 distinct SEL programs) found that children who participated in universal, curriculum-based SEL programs showed significant improvement in social competence, emotional competence, behavioural self-regulation, and early learning skills, and reduced behavioural and emotional challenges post-intervention compared to control group peers [29]. However, researchers have noted the currently limited understanding of specific program components related to positive outcomes $[26,29,30]$.

Recognising that child behaviour is highly influenced by teacher behaviour, many SEL programs aim to influence child outcomes by strengthening teachers' capacity and capability to implement evidence-based SEL practices with fidelity [31]. Yet, much research effort has focused on understanding the impact of these programs on children, with fewer evaluations addressing teacher-level outcomes. A recent meta-analysis evaluated the impact of teacher training (with and without a curriculum-based component) on both child and teacher outcomes, finding training was moderately effective at improving childcare quality, caregiver interaction skill, and children's social-emotional development [32]. The inclusion of explicit curricula alongside teacher training did not appear to be a significant moderator of program success. However, only five of the 19 studies in this review included a curriculum-based component. To our knowledge, there lacks a synthesis of research on the effectiveness of curriculum-based SEL interventions for teacher-level outcomes.

\subsection{Study Aim}

Closer examination of the impact of universal curriculum-based interventions (i.e., programs that include explicit SEL skill instruction and are delivered at the class-wide level) on a broad range of teacher-level outcomes is needed to understand the domains in which teachers benefit from SEL programs, and the pathways by which SEL programs can influence children's developmental trajectories [33]. The aim of the present paper, therefore, was to systematically examine the following research questions: (i) what type of teacher outcomes have been evaluated in studies examining universal curriculum-based SEL programs in ECEC settings, and what measures have been used to capture domains of teacher behaviour, practice, and wellbeing?; (ii) what does the literature reveal about the effectiveness of universal curriculum-based SEL programs in ECEC settings for teacher outcomes?; (iii) are certain program characteristics associated with program benefits?; and (iv) what are the methodological limitations of research examining the impact of universal curriculum-based SEL programs on teacher outcomes, and what recommendations can be made for future research?

\section{Methods}

\subsection{Search Strategy and Study Selection}

This systematic review was conducted in accordance with the Preferred Reporting Items for Systematic Reviews and Meta-Analyses (PRISMA) guidelines [34]. Three electronic databases, MEDLINE Complete, PsychINFO and ERIC were searched using combinations of the following key terms: intervention*, program*, curricul* and "early learning centre", "early learning center", preschool, preschool*, "pre school", "pre-school", childcare, "childcare", kinder*, "pre kindergarten", "pre-kindergarten", "pre-K", "pre K", "day care", daycare, "Head Start", HeadStart and social, emotion*, social-emotional, "SEL", "self-esteem", empathy, "emotional intelligence", "conflict resolution", "problem solving", resilien*, aggress*, anxi*, prevent", externali*, internali*, withdraw ${ }^{*}$ and educator*, teacher*, leader*. Additional articles were identified by scanning reference lists of included studies and relevant systematic reviews. The search aimed to identify peer-reviewed studies that evaluated the 
impact of universal, curriculum-based SEL programs in ECEC settings on teacher outcomes, published in English between 1999 and 2019. All database searches were carried out between July to August 2019.

\subsection{Inclusion and Exclusion Criteria}

Studies were assessed against the inclusion and exclusion criteria presented in Table 2.

Table 2. Inclusion and Exclusion Criteria.

\begin{tabular}{|c|c|c|}
\hline Category & Included & Excluded \\
\hline Research Design & $\begin{array}{l}\text { Randomised controlled trial, } \\
\text { quasi-experimental trial with a comparator } \\
\text { group (no limits applied on the type of } \\
\text { comparison group), or a single-group } \\
\text { pre-post design. }\end{array}$ & Single-case designs. \\
\hline Research Setting & $\begin{array}{l}\text { Centre-based Early Childhood Education and } \\
\text { Care settings, including kindergartens, } \\
\text { preschools, and child care services for } \\
\text { children from birth to } 6 \text { years of age. }\end{array}$ & $\begin{array}{l}\text { Family day care. } \\
\text { After school-hours care. }\end{array}$ \\
\hline \multirow{3}{*}{ Program Type } & $\begin{array}{l}\text { Curriculum-based Social and Emotional } \\
\text { Learning (SEL) program for preschool-aged } \\
\text { children that was delivered to all children } \\
\text { within the group (universal intervention). }\end{array}$ & $\begin{array}{l}\text { Classroom teacher/educator did not lead or } \\
\text { support delivery of the program. } \\
\text { Program targeted children experiencing } \\
\text { social, emotional, or behavioural difficulties, } \\
\text { or children diagnosed with a mental health } \\
\text { condition or developmental delay. }\end{array}$ \\
\hline & $\begin{array}{l}\text { SEL program addressed at least one of the } \\
\text { following competencies: self-awareness, } \\
\text { self-management, social awareness, } \\
\text { relationship skills, and responsible decision } \\
\text { making. }\end{array}$ & \\
\hline & $\begin{array}{l}\text { Program may include other components such } \\
\text { as teacher education, coaching, or } \\
\text { consultation in combination with the SEL } \\
\text { curriculum. }\end{array}$ & $\begin{array}{l}\text { Program focused on teacher education, } \\
\text { coaching, or consultation only, without a } \\
\text { curriculum component. }\end{array}$ \\
\hline Dependent Variable & $\begin{array}{l}\text { At least one educator-level outcome was } \\
\text { assessed following the intervention. This may } \\
\text { include, but was not limited to, teaching } \\
\text { quality, practices, or behaviour, the quality of } \\
\text { teacher-child interactions or teacher-child } \\
\text { relationships, or educator's own knowledge, } \\
\text { self-efficacy or social-emotional wellbeing. }\end{array}$ & $\begin{array}{l}\text { Did not report a teacher-level outcome post } \\
\text { intervention. }\end{array}$ \\
\hline Publication Status & $\begin{array}{l}\text { Published in English between January } 1999 \\
\text { and August } 2019 \text { and peer-reviewed. }\end{array}$ & $\begin{array}{l}\text { Unpublished reports and dissertations. } \\
\text { Articles published in lanugages other than } \\
\text { English. } \\
\text { Articles published prior to January } 1999 .\end{array}$ \\
\hline
\end{tabular}

\subsection{Review Procedures and Data Abstraction}

The systematic search identified 4205 articles after the removal of duplicates (Figure 1). All titles and abstracts were screened by one author (C.B.), with a second author (A.O.) independently co-screening $10 \%$ of the titles and abstracts; agreement for articles to be read in full was $100 \%$ after discussion. One hundred and ninety-nine papers were read in full, with 15 included in the review. Two articles that provided data relating to the same study were combined [35,36]. The following pre-specified data were extracted from each study: (i) ECEC setting; (ii) study design; (iii) sample size (number of teachers); (iv) teacher characteristics; (v) type of control group; (vi) SEL program; (vii) program components; (viii) teacher education component where relevant; (ix) outcome, outcome measure and informant (teacher, observer); and (x) findings, including effect sizes where reported by the author. 


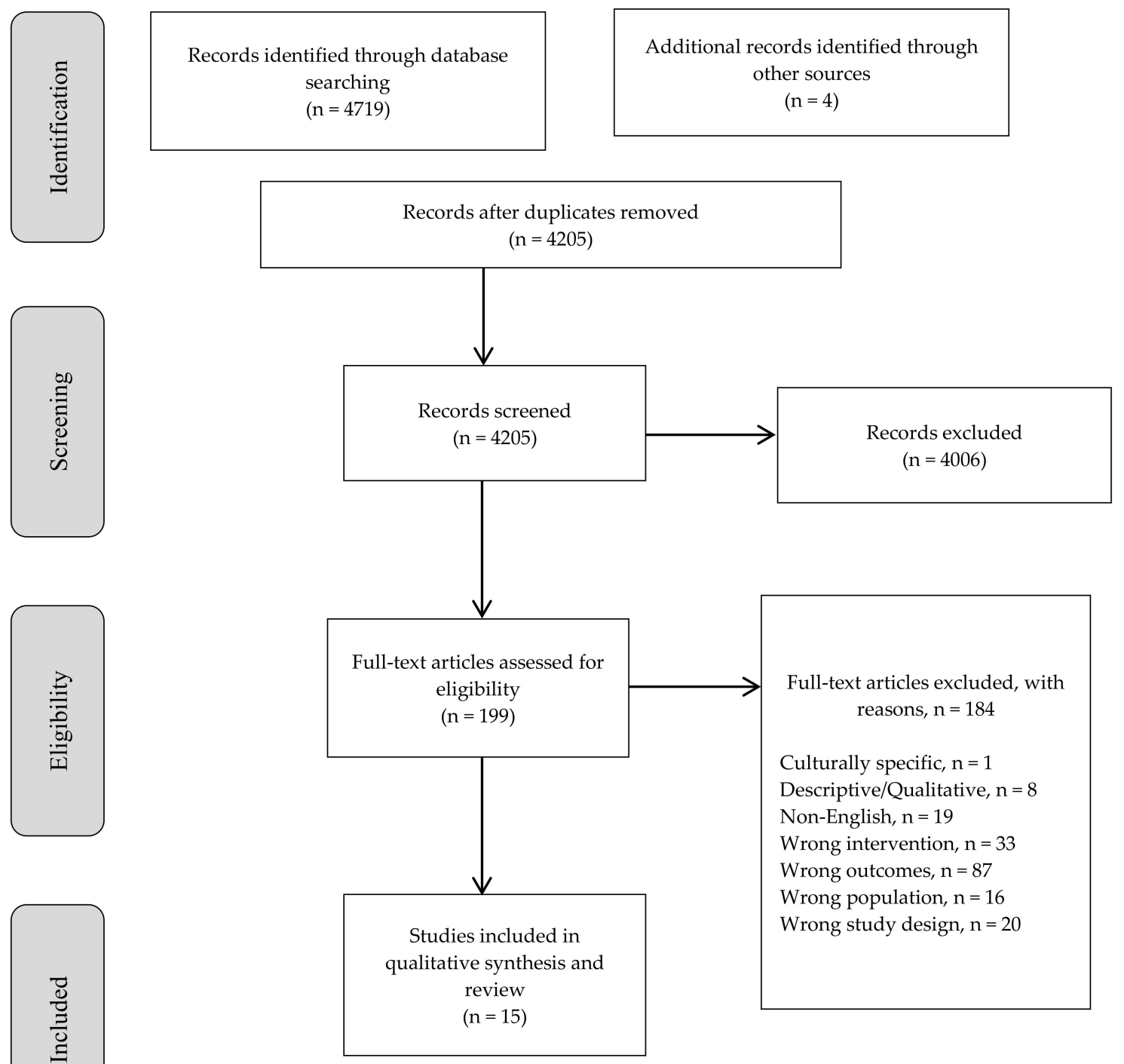

Figure 1. Flow diagram of studies included in review.

\subsection{Quality of Evidence}

Study quality was assessed against the Effective Public Health Practice Project (EPHPP) Quality Assessment Tool for quantitative studies with respect to selection bias, study design, confounders, blinding, data collection methods, withdrawals, dropouts, intervention integrity, and analyses [37]. This tool is suitable for randomised, non-randomised, and pre-post designs, and was utilised in our recent review examining the impact of curriculum-based SEL interventions on child outcomes [29]. Components were rated as strong, moderate or weak across each study, based on guidelines in the EPHPP Dictionary and an overall global quality rating was assigned. Studies were rated as strong when no weak ratings were recorded. Those with one weak rating were considered of moderate quality, and two or more weak ratings resulted in an overall weak rating.

\section{Results}

\subsection{Summary of Included Studies}

The pooled characteristics of the 15 studies included in this review are provided in Table 3 and detailed further in Table S1 in the Supplementary File. Table 4 summarizes the teacher-level 
outcomes, measures, and key findings from each paper. Ten studies were described as randomised or cluster-randomised controlled trials [36,38-47] and four quasi-experimental trials [48-51]. Settings included kindergarten [40,41,47], childcare [43,46], preschool [38,39,44,45,48-50], Head Start preschool or kindergarten (early childhood education services provided to low-income children and families in the United States) $[35,42,44,45,47,51]$ and early school grades $[40,44,47]$. In most studies, control group children participated in a business-as-usual ECEC curriculum $[n=13,35,38,39,41,43,44-51]$, with a smaller group of studies employing active controls including a literacy program [40], and a trust-based relational intervention with a relationship building course and daily activities [42]. Thirteen studies were conducted in the United States, and two in Turkey [38,50], and all were published in the last 15 years. A total of 736 teachers were captured by the studies in this review. One review reported their study was conducted in the kindergarten rooms of four low-income schools, including 327 children, however, did not specify the number of teacher participants [41]. Seven studies (46.7\%) were assessed as strong quality $[35,39,40,43,44,46,47]$, two $(13.3 \%)$ as moderate quality $[41,45]$, and six $(40.0 \%)$ as weak quality $[38,42,48-51]$ (Table 4$)$.

Table 3. Pooled Summary of the 15 Studies Investigating the Effect of Universal Curriculum-Based SEL Programs on Teacher-Level Outcomes.

\begin{tabular}{|c|c|}
\hline Characteristics & Sample $n$ (\% Where Applicable) \\
\hline \multicolumn{2}{|l|}{ Geographic Location } \\
\hline United States & $13(87 \%)$ \\
\hline Turkey & $2(13 \%)$ \\
\hline \multicolumn{2}{|l|}{ Date of Publication } \\
\hline 1999-2005 & $1(7 \%)$ \\
\hline 2006-2010 & $4(27 \%)$ \\
\hline 2011-2015 & $6(40 \%)$ \\
\hline 2015-2019 & $4(27 \%)$ \\
\hline \multicolumn{2}{|l|}{ Study Type } \\
\hline $\mathrm{RCT} / \mathrm{CRT}$ & 11 \\
\hline $\mathrm{QE}$ & 4 \\
\hline \multicolumn{2}{|l|}{ Sample Size (Teachers) } \\
\hline $0-10$ & $2(13 \%)$ \\
\hline $11-25$ & $2(13 \%)$ \\
\hline $26-50$ & $3(20 \%)$ \\
\hline $51-100$ & $4(27 \%)$ \\
\hline $101-150$ & $1(7 \%)$ \\
\hline $150+$ & $1(13 \%)$ \\
\hline Unclear & $2(7 \%)$ \\
\hline \multicolumn{2}{|l|}{ Intervention Leader } \\
\hline Teacher & $13(87 \%)$ \\
\hline Teacher and specialist/researcher & $2(13 \%)$ \\
\hline \multicolumn{2}{|l|}{ Program Duration (wk) } \\
\hline$<6$ & $1(7 \%)$ \\
\hline $6-12$ & $4(27 \%)$ \\
\hline $13-24$ & $3(20 \%)$ \\
\hline $25-36$ & $2(13 \%)$ \\
\hline$>36$ or embedded into pedagogy & $4(27 \%)$ \\
\hline Unclear & $1(7 \%)$ \\
\hline \multicolumn{2}{|l|}{ Professional Learning Support } \\
\hline Training/Workshops & 15 \\
\hline Classroom Visits & 4 \\
\hline Meeting/Consultation/Coaching & 5 \\
\hline \multicolumn{2}{|l|}{ Parent Engagement } \\
\hline Training Sessions/Workshops & $4(27 \%)$ \\
\hline Update/Bulletin/Newsletter & $5(33 \%)$ \\
\hline Not Described & $6(40 \%)$ \\
\hline
\end{tabular}


Table 4. Intervention Effects on Teacher-Level Outcomes.

\begin{tabular}{|c|c|c|c|c|c|}
\hline $\begin{array}{l}\text { First Author (Year) } \\
\text { Citation } \\
\end{array}$ & $\begin{array}{c}\text { Intervention } \\
\text { Sample Size Teacher (Children) } \\
\end{array}$ & Outcome (s) & Instrument (Informant) & Key Findings at Post-Intervention & Quality Assessment \\
\hline \multirow[t]{2}{*}{ Arda (2012) [38] } & \multirow[t]{2}{*}{$\begin{array}{c}\text { Preschool Promoting Alternative } \\
\text { THinking (Preschool PATHS) } \\
7 \text { (95) }\end{array}$} & $\begin{array}{l}\text { Teacher Behaviour and Management } \\
\text { Techniques: classroom structure and } \\
\text { management, discipline, emotional } \\
\text { communication and support, social } \\
\text { awareness and problem solving, } \\
\text { preventing misbehaviour }\end{array}$ & $\begin{array}{l}\text { The Teacher Style Rating Scale } \\
\text { (TSRS) (O) }\end{array}$ & $\begin{array}{l}\text { Intervention teachers outperformed control peers on } \\
\text { measures of discipline }(p<0.05) \text {, emotional } \\
\text { communication and support }(p<0.001) \text {, social } \\
\text { awareness and problem solving }(p<0.001) \text {, and } \\
\text { preventing misbehaviour }(p<0.001) \text {. Groups did not } \\
\text { differ on classroom structures and management. }\end{array}$ & \multirow[t]{2}{*}{ W } \\
\hline & & $\begin{array}{l}\text { Quality of the Classroom } \\
\text { Environment: assessment of child } \\
\text { behaviours, teacher } \\
\text { responsiveness/supports }\end{array}$ & $\begin{array}{l}\text { Classroom Atmosphere Rating } \\
\text { Scale (CARS) (O) }\end{array}$ & - $\quad$ Significant group differences on CARS $(p<0.001)$. & \\
\hline \multirow{4}{*}{ Barnett (2008) [39] } & \multirow{4}{*}{$\begin{array}{l}\text { Tools of the Mind } \\
18 \text { classrooms }(210)\end{array}$} & $\begin{array}{l}\text { Global Classroom Quality: space, } \\
\text { personal care routines, language and } \\
\text { reasoning, teacher-child interactions, } \\
\text { program structure, parent } \\
\text { involvement }\end{array}$ & $\begin{array}{l}\text { The Early Childhood } \\
\text { Environmental Rating } \\
\text { Scale-Revised (ECERS-R) (O) }\end{array}$ & $\begin{array}{l}\text { Intervention Group (IG) teachers scored significantly } \\
\text { higher than Control Group (CG) peers on ECERS-R } \\
(p=0.003) \text {. Significant differences reported for } \\
\text { activities }(p=0.004) \text { and language reasoning ( } p= \\
\text { 0.010) subscales, while interactions }(p=0.081) \\
\text { reached borderline significance. }\end{array}$ & \multirow{4}{*}{$\mathrm{S}$} \\
\hline & & $\begin{array}{l}\text { Literacy Environment and } \\
\text { Instruction }\end{array}$ & $\begin{array}{l}\text { Supports for Early Literacy } \\
\text { Assessment (SELA) (O) }\end{array}$ & $\begin{array}{l}\text { - IG teachers scored higher on the SELA }(p=0.001) \\
\text { compared to the CG. }\end{array}$ & \\
\hline & & Use of Scaffolding Techniques & $\begin{array}{l}\text { The Preschool Classroom } \\
\text { Implementation (PCI) Scale (O) }\end{array}$ & $\begin{array}{l}\text { IG teachers scored higher on the PCI }(p=0.002) \\
\text { compared to the CG. }\end{array}$ & \\
\hline & & $\begin{array}{l}\text { Emotional Climate, Classroom } \\
\text { Management, Instruction }\end{array}$ & $\begin{array}{l}\text { Classroom Assessment Scoring } \\
\text { System (CLASS) (O) }\end{array}$ & $\begin{array}{l}\text { TOOLS classrooms scored significantly higher than } \\
\text { CG on productivity }(p=0.042) \text { with a trend towards } \\
\text { higher levels of teacher sensitivity }(p=0.074) \text {. } \\
\text { Groups did not differ on positive classsoom climate, } \\
\text { negative climate, over-control, behaviour } \\
\text { management techniques, concept development, } \\
\text { learning formats and engagement and quality of } \\
\text { teacher feedback. }\end{array}$ & \\
\hline
\end{tabular}


Table 4. Cont

\begin{tabular}{|c|c|c|c|c|c|}
\hline $\begin{array}{l}\text { First Author (Year) } \\
\text { Citation }\end{array}$ & $\begin{array}{c}\text { Intervention } \\
\text { Sample Size Teacher (Children) }\end{array}$ & Outcome (s) & Instrument (Informant) & Key Findings at Post-Intervention & Quality Assessment \\
\hline Cappella (2015) [40] & $\begin{array}{c}\text { INSIGHTS } \\
120,60 \text { in K ( 16.57/class) }\end{array}$ & $\begin{array}{l}\text { Emotional Support and Classroom } \\
\text { Organisation }\end{array}$ & $\begin{array}{l}\text { Classroom Assessment Scoring } \\
\text { System (CLASS) (O) }\end{array}$ & $\begin{array}{l}\text { INSIGHTS teachers showed higher levels of } \\
\text { emotional support post intervention compared to } \\
\text { attention-control classrooms }(p<0.05, E S=0.30) \text {. } \\
\text { Treatment effect was moderated by grade, and more } \\
\text { pronounced for first grade rooms }(p<0.05, E S= \\
\text { 0.68). No differences between groups on level of } \\
\text { classroom organisation. }\end{array}$ & $\mathrm{s}$ \\
\hline \multirow{3}{*}{$\begin{array}{c}\text { Domitrovich (2009) [35] } \\
\text { Bierman (2014) [36] }\end{array}$} & \multirow{3}{*}{$\begin{array}{c}\text { Preschool PATHS } \\
84(246)\end{array}$} & $\begin{array}{l}\text { Emotional Support and Instructional } \\
\text { Support }\end{array}$ & $\begin{array}{l}\text { Classroom Assessment Scoring } \\
\text { System (CLASS) (O) }\end{array}$ & $\begin{array}{l}\text { CLASS showed moderate differences favouring the } \\
\text { IG for emotional support however this did not reach } \\
\text { statistical significance }(p=0.11, d=0.39,) \text {. Significant } \\
\text { effect on positive climate item }(d=0.61, p=0.04) \text { and } \\
\text { a borderline effect on teacher sensitivity }(d=0.58, p= \\
\text { 0.07) was reported. No group differences on negative } \\
\text { climate, over-control and behaviour management } \\
\text { subscales. A non-significant trend favouring IG was } \\
\text { reported for instructional support }(p=0.08, d=0.45) \text {. }\end{array}$ & \multirow{3}{*}{$\mathrm{s}$} \\
\hline & & $\begin{array}{l}\text { Teaching Style: positive discipline, } \\
\text { classroom management, positive } \\
\text { emotional climate }\end{array}$ & $\begin{array}{l}\text { The Teaching Style Rating Scale } \\
\text { (TSRS) (O) }\end{array}$ & $\begin{array}{l}\text { The TSRS showed IG improvement on the positive } \\
\text { emotional climate subscale (emotion expression, } \\
\text { emotion regulation and emotion modelling, } p= \\
\text { 0.05), and a significant intervention effect for } \\
\text { classroom management }(p=0.002) \text {. There was no } \\
\text { difference between groups on positive discipline, } \\
\text { however IG teachers scored higher on individual } \\
\text { item of proactive/preventive classroom management } \\
(p=0.001) \text {. }\end{array}$ & \\
\hline & & $\begin{array}{l}\text { Child-Directed Talk: directives, } \\
\text { questions, statements, } \\
\text { decontextualised talk, richness and } \\
\text { sensitivity of teacher's child centred } \\
\text { talk }\end{array}$ & $\begin{array}{l}\text { The Classroom Language and } \\
\text { Literacy Environment } \\
\text { Observation (CLEO) (O) }\end{array}$ & $\begin{array}{l}\text { IG teachers showed greater linguistic support, made } \\
\text { more statements }(p=0.001) \text {, asked more questions }(p \\
<0.001), \text { decontextualised utterances }(p=0.005) \text { and } \\
\text { engaged in richer and more sensitive talk with } \\
\text { children }(p=0.004) \text {. Effect sizes ranged from } d=0.67 \\
\text { to } d=0.89 \text {. No difference between groups } \\
\text { on directives. }\end{array}$ & \\
\hline
\end{tabular}


Table 4. Cont

\begin{tabular}{|c|c|c|c|c|c|}
\hline $\begin{array}{l}\text { First Author (Year) } \\
\text { Citation }\end{array}$ & $\begin{array}{c}\text { Intervention } \\
\text { Sample Size Teacher (Children) }\end{array}$ & Outcome (s) & Instrument (Informant) & Key Findings at Post-Intervention & Quality Assessment \\
\hline Fishbein (2016) [41] & $\begin{array}{l}\text { Preschool PATHS } \\
4 \text { schools (327) }\end{array}$ & $\begin{array}{l}\text { Student-Teacher Relationship: } \\
\text { closeness, conflict }\end{array}$ & $\begin{array}{c}\text { Student-Teacher Relationship } \\
\text { Scale }(\mathrm{T})\end{array}$ & $\begin{array}{l}\text { - Greater improvement in IG in Total Score }(p<0.001) \text {, } \\
\text { closeness }(p<0.001) \text { and conflict }(p<0.05) \text { subscales. }\end{array}$ & M \\
\hline Gunter (2012) [48] & $\begin{array}{l}\text { Strong Start Pre-K } \\
\quad 4(84)\end{array}$ & $\begin{array}{l}\text { Student-Teacher Relationship: } \\
\text { closeness, conflict, dependency }\end{array}$ & $\begin{array}{c}\text { Student-Teacher Relationship } \\
\text { Scale (T) }\end{array}$ & $\begin{array}{l}\text { Total score increased in both IGs, however only } \\
\text { reached statistical significance for the IG + booster } \\
\text { lesson group }(p<0.05, d=1.20) \text {. Both IG groups } \\
\text { showed decreased conflict }(p<0.05, \mathrm{~d}=0.43 \text { for } \\
\text { intervention and } 0.67 \text { for intervention }+ \text { booster), } \\
\text { while conflict in the CG increased. CG and } \\
\text { intervention + booster groups increased level of } \\
\text { closeness, with greatest improvement in the } \\
\text { intervention + booster condition }(p<0.05, d=1.35) \text {. } \\
\text { The IG group without boosters showed increased } \\
\text { dependency }(p<0.05, d=0.43) \text {, while IG + boosters } \\
\text { and CG showed decline. }\end{array}$ & W \\
\hline \multirow[t]{2}{*}{ Jackman (2019) [42] } & \multirow[t]{2}{*}{$\begin{array}{l}\text { OpenMind (OM) Curriculum } \\
27(262)\end{array}$} & Tendency to be mindful & $\begin{array}{l}\text { Five Facet Mindfulness } \\
\text { Questionnaire (FFMQ) (T) }\end{array}$ & $\begin{array}{l}\text { Groups differed on the Describe subscale of the } \\
\text { FFMQ. IG scores improved from baseline to } \\
\text { post-intervention while scores decreased for CG ( } p< \\
\text { 0.05). There was no difference between groups on } \\
\text { other subscales (observe, act with awareness, non- } \\
\text { judgmental, non-react). }\end{array}$ & \multirow[t]{2}{*}{$\mathrm{W}$} \\
\hline & & Perceived stress & Perceived Stress Scale-10 (T) & $\begin{array}{l}\text { - IG showed slight increase in teacher stress between } \\
\text { baseline }(M=20.33, S D=1.58) \text { and post-intervention } \\
(M=21.0, S D=2.24) \text {, while } C G \text { showed a slight } \\
\text { decrease between baseline }(M=21.14, S D=2.12) \\
\text { and post-intervention }(M=20.42, S D=2.30)\end{array}$ & \\
\hline
\end{tabular}


Table 4. Cont.

\begin{tabular}{|c|c|c|c|c|c|}
\hline $\begin{array}{l}\text { First Author (Year) } \\
\text { Citation }\end{array}$ & $\begin{array}{c}\text { Intervention } \\
\text { Sample Size Teacher (Children) }\end{array}$ & Outcome (s) & Instrument (Informant) & Key Findings at Post-Intervention & Quality Assessment \\
\hline \multirow[t]{2}{*}{ Landry (2014) [43] } & \multirow[t]{2}{*}{$\begin{array}{l}\text { Responsive Early Childhood } \\
\text { Curriculum (RECC) plus explicit } \\
\text { social-emotional activities } \\
65(542)\end{array}$} & $\begin{array}{l}\text { Teacher Behaviour: teacher } \\
\text { responsiveness and instruction }\end{array}$ & $\begin{array}{c}\text { Teacher Behaviour Rating Scale } \\
\text { (TBRS) (O) }\end{array}$ & $\begin{array}{l}\text { IG showed significantly greater improvement than controls } \\
\text { for the average of all TBRS subscales }(p<0.0001, E S=1.04) \text {. } \\
\text { The following subscales reached statistical significance: } \\
\text { classroom community }(p=0.009, E S=0.61) \text {, oral language ( } p= \\
0.011, E S=0.79) \text {, learning centres }(p \leq 0.0001, E S=1.74) \text {, book } \\
\text { reading }(p=0.001, E S=1.35), \text { written expression }(p=0.005, \\
E S=1.23), \text { print and letter }(p=0.0002, E S=1.35) \text {, and lesson } \\
\text { plans }(p<0.0001, E S=1.65) \text {. Groups did not differ on } \\
\text { subscales relating to sensitivity, discipline, phonological } \\
\text { awareness, mathematics, portfolios and team teaching. } \\
\text { Both RECC and RECC+ groups scored higher than controls, } \\
\text { and did not differ from each other. At post-intervention, total } \\
\text { score and } 8 / 13 \text { subscale scores for RECC and RECC+ groups } \\
\text { were between medium-low and medium-high quality. In CG, } \\
\text { only } 3 / 13 \text { subscales reached the medium-low quality rating. }\end{array}$ & \multirow[t]{2}{*}{$\mathrm{s}$} \\
\hline & & $\begin{array}{l}\text { Teacher-Child Relationship: } \\
\text { closeness, conflict }\end{array}$ & $\begin{array}{l}\text { Adult-Child Relationship Scale } \\
\text { (T) }\end{array}$ & $\begin{array}{l}\text { - Average closeness for RECC and RECC + was greater than } \\
\text { controls }(p=0.0065, E S=0.42) \text {. Teacher child conflict in RECC } \\
\text { and RECC+ was lower than controls }(p=0.011, E S=-0.49) \text {. }\end{array}$ & \\
\hline Lonigan (2015) [44] & $\begin{array}{l}\text { Preschool PATHS } \\
110(855)\end{array}$ & $\begin{array}{l}\text { Teacher Behaviour and Classroom } \\
\text { Characteristics }\end{array}$ & $\begin{array}{l}\text { Teacher Behaviour Rating Scale } \\
\text { (TBRS) (O) }\end{array}$ & $\begin{array}{l}\text { Teachers in the Explicit SEL group scored higher than controls } \\
\text { on the following classroom characteristics: classroom } \\
\text { community }(p<0.01, E S=0.73), \text { lesson planning }(p<0.001 \text {, } \\
E S=1.0) \text { and team teaching }(p<0.01, E S=0.77) \text {. The implicit } \\
\text { SEL group outperformed CG on the following subscales: } \\
\text { classroom community }(p<0.01, E S=0.85) \text {, discipline }(p< \\
0.05, E S=0.48) \text {, lesson planning }(p<0.01, E S=0.97) \text { and team } \\
\text { teaching reached borderline significance }(p<0.01, E S=0.49) \text {. } \\
\text { Explicit and implicit groups did not differ from each other. } \\
\text { No intervention effects were reported for teacher sensitivity or } \\
\text { learning centres. } \\
\text { On specific instructional activities, Explicit } S E L \text { group } \\
\text { outperformed CG on book reading }(p<0.01, E S=0.87) \text {, oral } \\
\text { language }(p<0.05, E S=0.57) \text { and math activities }(p<0.05, E S \\
=0.63) \text {. The implicit } S E L \text { group outperformed controls on } \\
\text { book reading }(p<0.001, E S=0.87), \text { oral language }(p<0.05, E S \\
=0.55), \text { phonological awareness }(p<0.05, E S=0.52) \text {, and } \\
\text { math activities }(p<0.01, E S=0.70) \text {. Explicit and implicit } S E L \\
\text { groups did not differ from each other. No intervention effects } \\
\text { were recorded for print activities or writing activities. }\end{array}$ & $\mathrm{s}$ \\
\hline
\end{tabular}


Table 4. Cont.

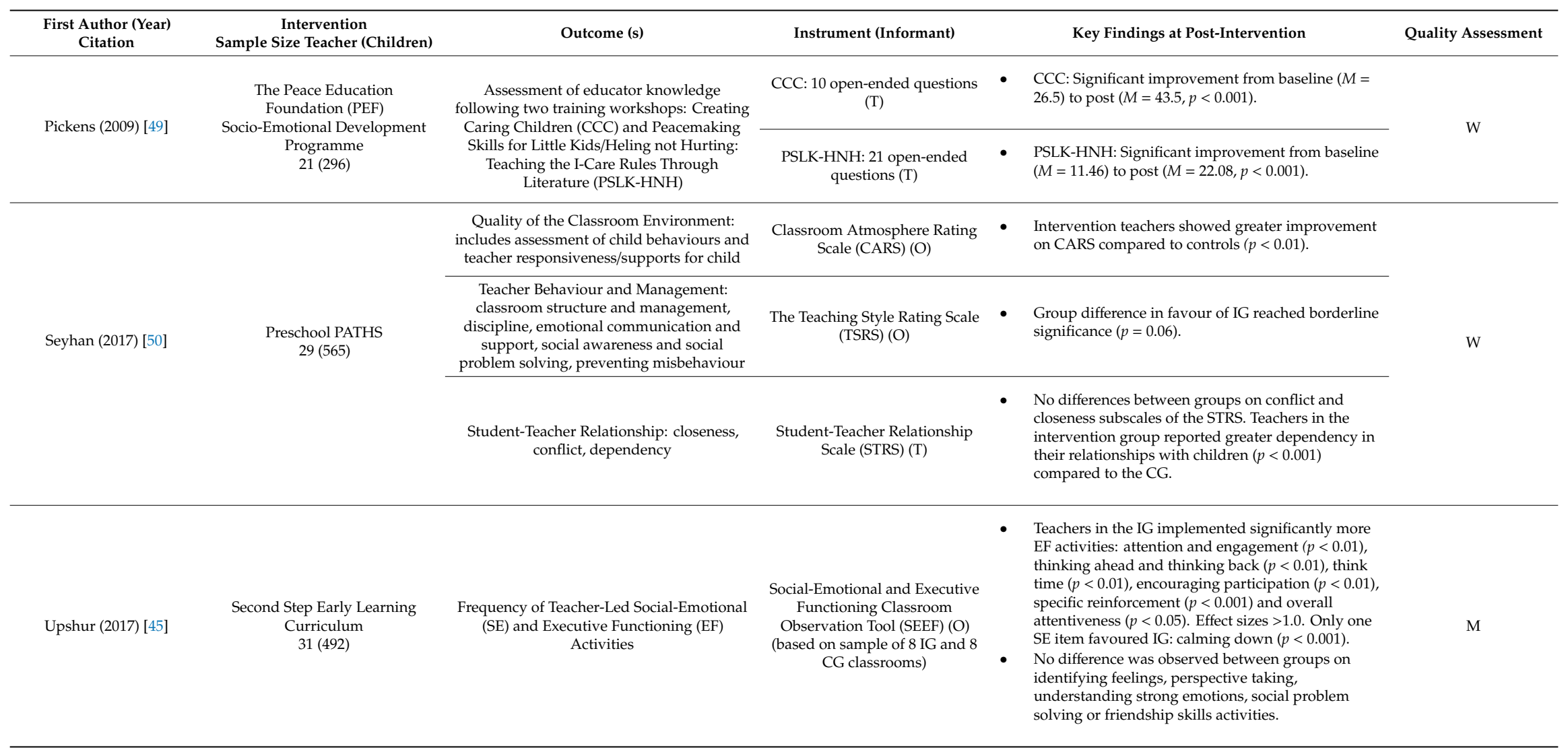


Table 4. Cont

\begin{tabular}{|c|c|c|c|c|c|}
\hline $\begin{array}{l}\text { First Author (Year) } \\
\text { Citation }\end{array}$ & $\begin{array}{c}\text { Intervention } \\
\text { Sample Size Teacher (Children) }\end{array}$ & Outcome (s) & Instrument (Informant) & Key Findings at Post-Intervention & Quality Assessment \\
\hline \multirow[t]{2}{*}{ Upshur (2013) [46] } & \multirow[t]{2}{*}{$\begin{array}{l}\text { Second Step Preschool/ } \\
\text { Kindergarten Social/Emotional } \\
\text { Learning curriculum } \\
56(341)\end{array}$} & $\begin{array}{l}\text { Interaction: discipline, general } \\
\text { supervision, staff-child interactions }\end{array}$ & $\begin{array}{l}\text { Early Childhood Environment } \\
\text { Rating Scale Revised } \\
\text { (ECERS-R), Interaction Scale (O) }\end{array}$ & $\begin{array}{l}\text { - In Year } 1 \text {, groups did not differ on any measures. } \\
\text { However, effect sizes favoured intervention } \\
\text { classrooms in the medium to high range for } \\
\text { ECERS-R interaction scale }(d=0.35) \text {, and ECERS-R } \\
\text { interaction items: discipline }(d=0.83 \text { ) and general } \\
\text { supervision }(d=0.32) \text {. In Year } 2, \text { IG showed greater } \\
\text { improvement in ECERS-R interaction scale }(p<0.05 \text {, } \\
d=1.81) \text { and discipline }(p<0.01, d=2.44) \text {. General } \\
\text { supervision }(p<0.10, d=1.78) \text { and staff-child } \\
\text { interactions }(p<0.10, d=1.49) \text { reached } \\
\text { borderline significance. } \\
\text { Results remained significant after adjustment } \\
\text { for covariates. }\end{array}$ & \multirow[t]{2}{*}{$S$} \\
\hline & & $\begin{array}{l}\text { Quality of Teacher Interaction Skill: } \\
\text { positive, punitive, permissive, } \\
\text { detached }\end{array}$ & $\begin{array}{l}\text { Caregiver Interaction Scale } \\
\text { (CIS) (O) }\end{array}$ & $\begin{array}{l}\text { - Change scores did not differ between groups in Year } \\
\text { 1. In Year 2, teacher interaction skills remained } \\
\text { stable for the IG and decreased significantly in CG ( } p \\
=0.03, d=1.74) \text {. }\end{array}$ & \\
\hline Vestal (2004) [51] & $\begin{array}{l}\text { I Can Problem Solve } \\
11(64)\end{array}$ & $\begin{array}{l}\text { Perceptions and Practices in Relation } \\
\text { to Conflict }\end{array}$ & ICPS dialogue $(\mathrm{T})$ & $\begin{array}{l}\text { Teachers used more ICPS dialogue after training and } \\
\text { decreased their non-ICPS dialogue }(p<0.05) \text {. ICPS } \\
\text { dialogue also increased from baseline to } \\
\text { post-intervention }(p<0.05) \text {. }\end{array}$ & W \\
\hline
\end{tabular}


Table 4. Cont

\begin{tabular}{|c|c|c|c|c|c|}
\hline $\begin{array}{l}\text { First Author (Year) } \\
\text { Citation }\end{array}$ & $\begin{array}{c}\text { Intervention } \\
\text { Sample Size Teacher (Children) }\end{array}$ & Outcome (s) & Instrument (Informant) & Key Findings at Post-Intervention & Quality Assessment \\
\hline \multirow{3}{*}{$\begin{array}{c}\text { Webster-Stratton (2008) } \\
\text { [47] }\end{array}$} & \multirow{3}{*}{$\begin{array}{c}\text { The Incredible Years Dina } \\
\text { Dinosaur Social Skills and } \\
\text { Problem Solving Curriculum } \\
153 \text { (1768) }\end{array}$} & $\begin{array}{l}\text { Teacher Behaviour: positive } \\
\text { reinforcement, critical statements, } \\
\text { amount of interaction with children }\end{array}$ & $\begin{array}{l}\text { Multiple Option Observation } \\
\text { System for Experimental } \\
\text { Studies (MOOSES) (O) }\end{array}$ & $\begin{array}{l}\text { Based on MOOSES, a reduction in critical statements } \\
\text { favoured IG. The more critical the teacher was } \\
\text { initially, the more the score improved at post. No } \\
\text { other constructs reported significant effects. }\end{array}$ & \multirow{3}{*}{$\mathrm{s}$} \\
\hline & & $\begin{array}{l}\text { Teaching Style and Classroom } \\
\text { Management: harsh/critical, } \\
\text { inconsistent/permissive, } \\
\text { warm/affectionate, social/emotional } \\
\text { teaching, effective discipline }\end{array}$ & $\begin{array}{l}\text { Teacher Coder Impressions } \\
\text { Inventory (TCI) (O) }\end{array}$ & $\begin{array}{l}\text { After controlling for covariates, IG teachers were less } \\
\text { harsh/critical (ES }=0.67) \text {, and } \\
\text { inconsistent/permissive }(E S=0.63) \text {, more } \\
\text { warm/affectionate }(E S=0.51) \text { and placed more } \\
\text { emphasis on social-emotional teaching }(E S=0.96) \text {. } \\
\text { Main effects for effective discipline did not emerge, } \\
\text { but intervention effect depended on the grade of the } \\
\text { teacher: Kindergarten and Grade } 1 \text { teachers showed } \\
\text { higher levels of effective discipline than Head } \\
\text { Start teachers. }\end{array}$ & \\
\hline & & $\begin{array}{l}\text { Quality of the Classroom } \\
\text { Atmosphere: includes assessment of } \\
\text { child behaviours and teacher's } \\
\text { classroom management }\end{array}$ & $\begin{array}{l}\text { Classroom Atmosphere } \\
\text { Measure }(\mathrm{O})\end{array}$ & $\begin{array}{l}\text { - Greater improvement in IG's classroom atmosphere } \\
\text { compared to } C G(E S=1.03) \text {. }\end{array}$ & \\
\hline
\end{tabular}

Note: $\mathrm{CG}=$ Control Group, $\mathrm{IG}=$ Intervention Group, $\mathrm{M}=$ Moderate, $\mathrm{O}=$ Observer, $\mathrm{S}=$ Strong, $\mathrm{T}=$ Teacher, $\mathrm{W}=$ Weak. 


\subsection{SEL Program Characteristics}

Ten SEL programs were evaluated within the included studies; five papers examined Preschool Promoting Alternative THinking Strategies (Preschool PATHS) [35,38,41,44,50], two the Second Step Preschool/Kindergarten Social/Emotional Learning Curriculum and Second Step Early Learning Curriculum $[45,46]$ and one each the Incredible Years Dina Dinosaur Social Skills and Problem Solving Curriculum (Incredible Years Curriculum) [47], Tools of the Mind [39], INSIGHTS [40], Strong Start Pre-K [48], OpenMind [42], Responsive Early Childhood Curriculum, plus explicit SEL activities (RECC+) [43], I Can Problem Solve [51] and the Peace Education Foundation Curriculum [49]. INSIGHTS [40] was delivered by a trained facilitator and the Incredible Years Curriculum [47] by the lead researcher, both in partnership with the classroom teacher. All other programs were led by the classroom teacher. With the exception of Tools of the Mind [39] and OpenMind [42], which embedded SEL activities into the curriculum, studies included explicit SEL lessons or activities. As shown in Table 3, most programs provided early childhood educators with training and support during implementation.

\subsection{Teacher-Level Outcomes and Measures}

The studies captured in this review examined the following teacher-level outcomes: (i) teaching practice and behaviour, including teacher-child interactions; (ii) teacher-child relationship quality; (iii) teachers' social-emotional wellbeing; and (iv) teacher knowledge of SEL. Eleven studies examined the impact of SEL programs on teaching practice and behaviour, using observational assessments. Measures included the Teacher Style Rating Scale (TSRS) [35,38,50], an assessment of positive discipline, classroom structure and management, emotional communication and support, social awareness and social problem solving; the Classroom Assessment Scoring System (CLASS) $[35,39,40]$ to gauge emotional, organisational and instructional interactions within the classroom; the Teacher Behaviour Rating Scale [43,44], with subscales measuring the quantity and quality of specific teaching behaviours; the Caregiver Interactions Scale [46] addressing the quality of teacher-child interactions across positive, punitive, permissive and detached domains; and the Multiple Option Observation System for Experimental Studies to code teacher-focused behaviours including positive reinforcement/praise, critical statements, and the amount of interaction/involvement with children [47]. The Teacher Coder Impressions Inventory was included in one study to evaluate teaching style across five scales: harsh/critical, inconsistent/permissive, warm/affectionate, social-emotional teaching and effective discipline [47].

An overall assessment of classroom quality (including space, personal care routines, language and reasoning, interactions, program structure and parent involvement) using the Early Childhood Environmental Rating Scale-Revised was included in two studies [39,46], and classroom environment quality using the Classroom Atmosphere Rating Scale in another two [38,50]. This measure includes assessment of both teacher responsiveness and supports, and a global measure of child behaviour. Four studies examined specific teaching practices, including the use of scaffolding with the Preschool Classroom Observation Scale [39], literacy instruction with the Supports for Early Literacy Assessment [39], child-directed talk with the Classroom Language and Literacy Environmental Observation [35], and frequency of social-emotional and executive functioning activities using the Social-Emotional and Executive Functioning Classroom Observation Tool [45].

Four studies included a measure of teacher-child relationship quality based on teacher report. Three used the Student-Teacher Relationship Scale [41,48,50], and one the Adult-Child Relationship Scale [43]. Only one author measured teacher's own social-emotional wellbeing, examining stress levels using the Perceived Stress Scale, and tendency to be mindful with the Five Facet Mindfulness Questionnaire [42]. Finally, two authors assessed teacher knowledge of SEL techniques, including open-ended test questions to assess teachers' understanding of topics, activities and skill before and after taking part in the Creating Caring Children and Peacemaking Skills for Little Kids/Helping Not 
Hurting training sessions [49] and teacher dialogue before and after a 13-session college course to support implementation of I Can Problem Solve in Head Start classrooms [51].

\subsection{Effects of SEL Intervention on Teaching Practice and Behaviours}

The four studies that examined the impact of the Preschool PATHS curriculum on teaching practice and behaviour reported improvement post intervention [35,38,44,50]. Intervention group teachers outperformed controls who did not participate in a SEL intervention on a measure capturing effective discipline, emotional communication and support, social awareness and problem solving, and behaviour management in Arda and Ocak [38], and a trend towards improvement on the same measure was observed in another study of 29 Turkish teachers [50], who also found enhanced classroom environment quality in favour of intervention group participants.

The emotional climate (assessed as emotion expression, emotion regulation and emotional modelling) and effective classroom management subscales of the TSRS suggested greater improvement in teachers who delivered Preschool PATHS at post-intervention compared to a comparison group in Domitrovich and colleague's study [35]. An intervention effect did not emerge for positive discipline, however intervention teachers scored significantly higher on the proactive/preventive classroom management subscale. PATHS teachers also demonstrated greater emotional support on the CLASS measure. However, this did not reach statistical significance. Analyses of individual subscales however suggested a significant and moderate intervention effect on positive climate, and a borderline significant effect on teacher sensitivity. Improvement in the instructional support scale also reached borderline significance. Groups in this study did not differ on measures of productivity, quality of feedback, concept development or instructional learning formats. Teachers did, however, make more statements and ask more questions than control group peers based on the Classroom Language and Literacy Environmental Observation measure.

Bierman et al. [36] conducted follow-up assessments at one year post-intervention for $82 \%$ of the teachers who implemented the Preschool PATHS curriculum in Domitrovich et al.'s [35] study. Teachers who had delivered Preschool PATHS rated higher on the emotional climate scale and all subscales (emotional expression, emotion regulation, and emotional modelling) of the TSRS and the emotional support scale of the CLASS. Intervention effects favouring PATHS teachers were also reported for the positive discipline scale of the TSRS. The classroom management scale reached borderline significance, however there were no meaningful group differences for instructional support assessed by the CLASS measure. Teachers who participated in PATHS also asked children more general questions one year post-intervention, with differences in the number of statements, contextualized talk, ratings of sensitivity and richness of talk appearing marginally significant in favour of the intervention group.

In a cluster-randomised controlled trial, Lonigan et al. [44] compared a literacy and math-focused preschool curriculum including Preschool PATHS lessons (explicit SEL) and a version where teachers were provided with professional development and guidance on behaviour management but these skills were not the focus of any specific classroom activity (implicit SEL), to a business-as-usual condition. Observations showed that both intervention groups (with and without explicit SEL curricula) made significant improvements in classroom community, use of lesson plans, and team teaching compared to controls, albeit the two intervention groups did not differ significantly from each other on these outcomes. The curricula without explicit SEL lessons appeared to improve teachers' use of effective discipline strategies, however this did not emerge for the explicit SEL group. The two SEL groups did not differ from controls on measures of teacher sensitivity or learning centres (the provision of engaging and age-appropriate materials linked to learning themes).

Using a similar research design, another study compared the Responsive Early Childhood Curriculum with and without explicit social-emotional classroom activities to a control group receiving no intervention. Childcare teachers in both intervention groups (with and without the explicit SEL component) outperformed comparison group peers on a measure of teacher responsiveness and 
instruction. The inclusion of explicit SEL activities did not appear to strengthen the intervention effect [43].

Barnett et al. [39] found teachers who delivered Tools of the Mind curriculum demonstrated significantly higher productivity (management of instructional time and routines) compared with control group teachers, with assessment of teacher sensitivity (responsiveness and offering a secure base to children) reaching borderline significance. Teachers also used more scaffolding techniques than controls, provided a richer literacy learning environment, and scored higher on an overall assessment of classroom quality using the Early Childhood Environmental Rating Scale - Revised. Results did not indicate differences between groups on positive, negative, or over-controlling classroom climate, behaviour management techniques, concept development, learning engagement, or quality of teacher feedback. Similarly, teachers who delivered INSIGHTS to Kindergarten and Grade 1 classrooms in the United States offered children higher levels of emotional support post-intervention, after controlling for pre-test score and covariates, compared with attention-control group teachers who provided a literacy program. These effects were moderated by classroom level; the impact appeared more pronounced for first grade teachers and least pronounced for kindergarten educators. Levels of classroom organisation did not differ between groups at post-intervention [40].

The Incredible Years Curriculum, delivered in conjunction with the Incredible Years Teacher Classroom Management Program, led to positive improvement in teacher behaviour in a randomised controlled trial of 153 teachers and 1768 children [47]. Multi-level modelling suggested that intervention group teachers became less harsh/critical and inconsistent/permissive, appeared more warm/affectionate, and placed greater emphasis on social-emotional teaching. Improvement in effective discipline appeared to depend on setting; kindergarten and Grade 1 teachers showed greater improvement than Head Start teachers [47]. Similarly, intervention teachers used fewer critical statements with children, with the teachers observed to be the most critical at baseline making the greatest improvement. Intervention effects were not observed for measures of teacher involvement or levels of teacher praise.

Upshur, Wenz-Gross, and Reed [46] evaluated the Second Step curriculum across two annual cohorts in community childcare centres. Intervention teachers in the first cohort did not appear to differ from control peers in the quality of their interactions with children. The second cohort however showed greater improvement in teacher-child interaction skill and effective discipline. These effects remained significant in an adjusted model accounting for covariates and nesting of children within classrooms. Non-significant trends with large effects favouring the intervention classrooms were reported for general supervision and staff-child interactions. The Second Step Early Learning Curriculum combined instruction and activities to improve children's social-emotional competence and executive functioning. Intervention group teachers implemented significantly more executive functioning activities at post-intervention than control peers, however only one social-emotional outcome (calming down) favoured the intervention group. There were no differences between conditions on the frequency of other SEL activities, including identifying feelings, perspective taking, helping children to understand strong emotions, social problem solving or friendship skills [45].

\subsection{Effects of SEL Intervention on Teacher-Child Relationship Quality}

Four studies included a teacher-rated assessment of teacher-child relationship quality, a construct closely related to teacher-child interactions [18], with mixed effects reported. Participation in Preschool PATHS did not lead to improvement in teacher-child conflict or closeness, but was associated with increased dependency (an overreliance on the teacher as a source of support) in one study [50]. In another that compared teachers who delivered the Strong Start curricula, a group who delivered Strong Start and two booster lessons, and a control group, all three conditions showed improvement in teacher-child closeness at post-intervention. Further, teachers in the intervention group who did not receive booster lessons reported significantly greater levels of dependency in their relationships with children, while the group with boosters and control peers reported a decrease. The intervention 
was, however, associated with decreased levels of teacher-child conflict, while conflict in the control group increased. This improvement was most pronounced for teachers that delivered the curricula with two booster lessons [48]. In a randomised controlled trial of Preschool PATHS in a kindergarten setting, intervention teachers reported greater improvement in overall relationship quality, conflict and closeness compared to the control group, however closeness did not remain significant in a propensity score-matched sample controlling for baseline differences [41]. Likewise, Landry et al. [43] found teachers of children aged 2 to 3 years who participated in the Responsive Early Childhood Curriculum with and without an explicit SEL component reported greater improvement in closeness and reduced conflict with children compared to controls.

\subsection{Effects of SEL Intervention on Social-Emotional Wellbeing}

Only one study considered the impact of SEL on teachers' social-emotional wellbeing. Jackman and colleagues [42] evaluated the OpenMind curriculum, including child, teacher and parent components. Teachers attended a five-day training course focused on meditation, were requested to meditate for 20 minutes per day and facilitate daily practices with children in their classrooms. Authors revealed that intervention teachers were better able to describe their feelings compared with controls, albeit there was no effect on other aspects of dispositional mindfulness: observing, acting with awareness, non-judging and non-reactivity. Results suggested a slight increase in teacher stress in the intervention group, and a slight decrease in the control group; however, this did not reach statistical significance.

\subsection{Effects of SEL Intervention on Educator Knowledge of Social-Emotional Learning}

Two studies reported improvement in teacher knowledge of SEL following training that accompanied a classroom curriculum. Teachers who attended the Creating Caring Children and Peacemaking Skills for Little Kids/Helping Not Hurting training as part of the Peace Education Foundation program exhibited significant improvement in their knowledge of program concepts between pre- and post-assessment [49]. Similarly, teachers who attended a 13-session college course to support implementation of I Can Problem Solve showed significant improvement in conflict resolution practices [51].

\section{Discussion}

The social-emotional skills that emerge during early childhood are vital for later social-emotional competence [52]. An established body of research evidence highlights the benefits of high-quality ECEC for children's healthy development [1,53-55], and there is increasing focus on programmatic approaches to encourage educators to intentionally foster children's social and emotional skill growth within the Early Learning environment. Evaluations of SEL programs that target both educator behaviour and child outcomes in ECEC settings suggest benefits for children across developmental domains. Less is known however about the effects of these interventions on teaching quality and practice. To our knowledge, this is the first systematic review to consider the effectiveness of universal curriculum-based SEL interventions in ECEC settings for educator-level outcomes.

\subsection{Teacher-Level Outcomes Evaluated in SEL Research}

Studies examined the impact of SEL programs on teaching quality, teaching practice and behaviour, teacher-child interactions and relationship quality. Only one study included a pre-post measure of teacher wellbeing [42], and two assessed educator knowledge [49,51]. Teachers' beliefs, knowledge, experiences, self-efficacy, mental health, and social-emotional competence directly influence their ability to support children's social-emotional development $[10,11,13-15,56]$. However, the impact of SEL programs on these personal attributes could not be determined. 


\subsection{Effectiveness of Universal SEL Programs for Teacher-Level Outcomes}

Most evaluations captured in this review reported improvement in at least one aspect of teaching practice as a result of the SEL program. Findings, however, varied substantially across studies and outcome measures. The following programs appeared to strengthen teachers' emotional support, sensitivity, responsivity, or capacity to create a positive classroom climate: Preschool PATHS [35,38], the Incredible Years Curriculum [47], Tools of the Mind [39], INSIGHTS [40], and RECC+ [43]. This is an interesting finding given the importance of emotional support within ECEC settings. High-quality emotional interactions have been associated with children's social skills, after adjusting for prior skills, child, family, and program characteristics [57], and higher levels of social competence [58]. Researchers also suggest that emotional support may benefit behavioural engagement, which in turn encourages pre-academic skills [2,59].

Responsive caregiving, an important aspect of emotional support, was captured in several studies. Responsivity encompasses educators' ability to read and respond to children's cues, and individualize their teaching style to child need [60]. Developmental theory posits that responsivity can encourage attachment between a caregiver and child that fosters positive emotional, social, and cognitive development [61]. However, researchers suggest there can be a tendency for infrequent responsive and cognitively challenging conversations between teachers and children in early childhood settings, especially for children experiencing disadvantage, with some studies reporting that preschool programmes serving low-income communities appear to offer limited opportunities for responsive teacher-child interactions $[19,20]$. The improvement in observed emotional support from teachers who participated in SEL programs is therefore a promising finding.

Several authors also reported improvement in teachers' use of positive classroom management and discipline strategies at post intervention $[35,36,38,44,46,47]$. Behaviour guidance within the early years classroom can strengthen children's self-regulation. For example, effective classroom management in kindergarten settings has been associated with children's behavioural and cognitive self-control, behavioural engagement, and reduced duration of off-task behaviour [62]. These findings highlight a potential pathway between SEL intervention and improved child outcomes via teaching practices that promote children's cooperation and prosocial behaviour.

While positive intervention effects were reported across several studies, it is important to recognise the time and effort required to participate in professional learning and implement SEL with fidelity may also have a detrimental effect for some educators. For example, Jackman and colleagues found educators who participated in the OpenMind curriculum reported increased stress levels at post-intervention compared to controls, though this did not reach statistical significance [42]. Lonigan et al. [44] compared a lesson-based SEL curriculum (Preschool PATHS) with an implicit model, reporting improvement in teaching practice for educators in both conditions; however, no difference between the implicit and explicit versions. Similarly, Landry et al. [43] found explicit SEL activities did not appear to offer additional benefit beyond a responsive early childhood curriculum. Continued investigation of the benefits of explicit instruction in combination with implicit approaches, for both children and teachers, is needed to ensure educator time and effort is warranted.

\subsection{Program Characteristics Associated with Improvement in Teacher-Level Outcomes}

SEL programs shared a common goal to strengthen educators' capability to foster children's social-emotional skills through explicit and active instruction, modelling, reinforcement, and practice, albeit differing in several respects including the SEL competencies targeted, program intensity and duration, and the extent to which educators' own social-emotional wellbeing was addressed. Based on the research currently available, it is difficult to specify SEL program characteristics associated with program success.

A common feature across studies, however, was the professional learning support offered to educators. Many SEL programs paired comprehensive teacher education with regular consultation focused on educators' knowledge of strengthening social-emotional development in the preschool 
setting. Research suggests behaviour change in early years settings is most likely when specific training is combined with on-the-job coaching, feedback on observed performance, assistance with planning and implementation, and support with challenges and decision-making [63]. It is possible the sustained support offered as part of SEL interventions prompted teachers' continued awareness of their teaching practices and interactions, and strengthened their ability to effectively guide children's attention and behaviour. Specifically, the specialized training prior to SEL intervention may have strengthened teachers' attitudes, knowledge, and skills by allowing for rehearsal (e.g. through practice, role play) and individualized feedback [64], while the coaching and ongoing support may have increased the likelihood that these skills were embedded into educators' everyday practice.

\subsection{Methodological Limitations in the Evidence and Future Recommendations}

There are several limitations to the current evidence base that should be acknowledged in interpreting the findings. While many studies were strengthened by the use of controlled designs, validated scales to measure teacher-level outcomes and moderate to high study quality, they varied in the teacher-level outcomes explored, the type of SEL intervention examined, and the form and extent of professional learning offered. Variability in methodologies and measures is indicative of the multi-faceted nature of educational research, and creates complexity when comparing and integrating results across studies, particularly with regards to identifying components of SEL intervention that offer particular benefit for teacher outcomes. The CASEL framework which guided this review emphasizes the importance of a systemic approach to children's SEL, with integrated and coordinated supports and policies across classroom, service, family, and community levels [23]. It was not possible to ascertain the broader supports offered to educators within the included studies, which may have influenced intervention effects.

Furthermore, it is possible that teacher-level outcomes may mediate or moderate teachers' ability to effectively deliver the SEL curriculum. Continued exploration of the linkages between: (i) curriculum-based SEL programs, (ii) professional development and supports, (iii) teacher-level outcomes, and (iv) child outcomes is needed to understand the active ingredients and core components of successful programs. Additionally, investigation into the relative importance and effectiveness of teacher education, SEL curriculum, and the combination of both on teacher and child outcomes would benefit future SEL program development.

Finally, there lacks evidence of the sustainability of improvements in teacher outcomes over time. Only one study included a follow-up assessment $[35,36]$ and the potential benefits of SEL curriculum for ongoing teaching practice is unknown. It is vital that researchers utilise longitudinal methods to better understand the components of SEL program design that lead to social-emotional skill growth, for both teachers and children.

\subsection{Strengths and Limitations of the Current Review}

This review is strengthened by the clearly focused and pre-specified research questions, a thorough and systematic literature search and screening process, detailed data extraction, and assessment of study quality using a validated quality assessment tool. However, the exclusion of unpublished literature and dissertations, studies that were reported in languages other than English, and studies published prior to 1999 means it is possible relevant studies have been missed, potentially introducing bias into the results. Furthermore, due to the heterogeneity in study designs and outcome measures, global effect sizes were not calculated and the review relied on a non-qualitative analysis. As more research becomes available, statistical synthesis of effect sizes across domains of teaching practice, behaviour, and wellbeing may provide further insight [65]. Finally, while the randomised controlled trials captured in this review offer high levels of internal validity, the ecological validity of findings in everyday practice may be limited. 


\section{Conclusions}

The findings of this systematic review suggest that universal curriculum-based SEL programs in ECEC settings may strengthen teaching practice and behaviour, particularly the provision of responsive and nurturing teacher-child interactions and effective management of the classroom environment. However, several gaps in knowledge exist. Data were insufficient to ascertain whether participation in SEL programs improved teacher-child relationship quality or teachers' knowledge, self-efficacy, or social-emotional wellbeing. Further, there was no rigorous evidence of the sustainability of outcomes over time. Due to the diversity in the type of SEL programs and outcome measures captured, it was difficult to identify common features of SEL programs associated with improved teacher-level outcomes. Continued investigation of differential intervention effects (e.g., do certain programs benefit certain groups of educators), the impact of systemic SEL policies and approaches in addition to classroom curricula, and the association between implementation fidelity and both teacher and child outcomes is needed. This review adds to a growing body of SEL research in ECEC settings by exploring the potential pathways between curriculum-based SEL approaches and domains of teaching practice which are critical for children's developmental trajectories.

Supplementary Materials: The following are available online at http://www.mdpi.com/1660-4601/17/3/1049/s1, Table S1: Characteristics of Included Studies.

Author Contributions: Conceptualization, C.B., H.S., and A.O.; Methodology, C.B., H.S., and A.O.; Software, N/A; Validation, A.O.; Formal Analysis, N/A; Investigation C.B. and A.O.; Resources, N/A; Data Curation, C.B. and A.O.; Writing-Original Draft Preparation, C.B. and H.S.; Writing-Review \& Editing, C.B., H.S., A.O., H.M., A.M., H.B. (Heidi Bergmeier), K.J., A.N., and H.B. (Helen Barrett); Visualization, C.B.; Supervision, H.S., H.M., A.M., A.N.; Project Administration, C.B. and H.S.; Funding Acquisition, N/A. All authors have read and agreed to the published version of the manuscript.

Funding: This research received no external funding.

Conflicts of Interest: The authors declare no conflicts of interest.

\section{References}

1. Camilli, G.; Vargas, S.; Ryan, S.; Barnett, W.S. Meta-Analysis of the Effects of Early Education Interventions on Cognitive and Social Development. Teach. Coll. Rec. 2010, 112, 579-620.

2. Burchinal, M.; Howes, C.; Pianta, R.; Bryant, D.; Early, D.; Clifford, R.; Barbarin, O. Predicting Child Outcomes at the End of Kindergarten from the Quality of Pre-Kindergarten Teacher-Child Interactions and Instruction. Appl. Dev. Sci. 2008, 12, 140-153. [CrossRef]

3. Howes, C.; Burchinal, M.; Pianta, R.; Bryant, D.; Early, D.; Clifford, R.; Barbarin, O. Ready to learn? Children's pre-academic achievement in pre-Kindergarten programs. Early Child. Res. Q. 2008, 23, 27-50. [CrossRef]

4. Mashburn, A.J. Quality of social and physical environments in preschools and children's development of academic, language, and literacy skills. Appl. Dev. Sci. 2008, 12, 113-127. [CrossRef]

5. Ponitz, C.C.; Rimm-Kaufman, S.E.; Grimm, K.J.; Curby, T.W. Kindergarten classroom quality, behavioral engagement, and reading achievement. Sch. Psychol. Rev. 2009, 38, 102-120.

6. Duncan, G.J.; Sojourner, A.J. Can intensive early childhood intervention programs eliminate income-based cognitive and achievement gaps? J. Hum. Resour. 2013, 48, 945-968. [PubMed]

7. Loeb, S.; Fuller, B.; Kagan, S.L.; Carrol, B. Child Care in Poor Communities: Early Learning Effects of Type, Quality, and Stability. Child Dev. 2004, 75, 47-65. [CrossRef]

8. Magnuson, K.A.; Meyers, M.K.; Ruhm, C.J.; Waldfogel, J. Inequality in Preschool Education and School Readiness. Am. Educ. Res. J. 2004, 41, 115-157. [CrossRef]

9. Schleicher, A. Helping our Youngest to Learn and Grow: Policies for Early Learning; OECD Publishing: Paris, France, 2019.

10. Tournaki, N.; Podell, D.M. The impact of student characteristics and teacher efficacy on teachers' predictions of student success. Teach. Teach. Educ. 2005, 21, 299-314. [CrossRef]

11. Goroshit, M.; Hen, M. Does Emotional Self-efficacy Predict Teachers' Self-efficacy and Empathy? J. Educ. Train. Stud. 2014, 2, 26-32. [CrossRef] 
12. McMullen, M.B. Characteristics of teachers who talk the DAP talk and walk the DAP walk. J. Res. Child. Educ. 1999, 13, 216-230. [CrossRef]

13. Fantuzzo, J.; Perlman, S.; Sproul, F.; Minney, A.; Perry, M.A.; Li, F. Making visible teacher reports of their teaching experiences: The early childhood teacher experiences scale. Psychol. Sch. 2012, 49, 194-205. [CrossRef]

14. Jennings, P.A.; Greenberg, M.T. The prosocial classroom: Teacher social and emotional competence in relation to student and classroom outcomes. Rev. Educ. Res. 2009, 79, 491-525. [CrossRef]

15. Zinsser, K.M.; Bailey, C.S.; Curby, T.W.; Denham, S.A.; Bassett, H.H. Exploring the predictable classroom: Preschool teacher stress, emotional supportiveness, and students' social-emotional behavior in private and Head Start classrooms. NHSA Dialog. 2013, 16, 90-108.

16. Early, D.M.; Maxwell, K.L.; Ponder, B.D.; Pan, Y. Improving teacher-child interactions: A randomized controlled trial of Making the Most of Classroom Interactions and my teaching partner professional development models. Early Child. Res. Q. 2017, 38, 57-70. [CrossRef]

17. Early, D.M.; Maxwell, K.L.; Burchinal, M.; Bryant, D.; Cai, K.; Clifford, R.M.; Henry, G.T.; Peisner-Feinberg, E.; Vandergrift, N.; Alva, S.; et al. Teachers' education, classroom quality, and young children's academic skills: Results from seven studies of preschool programs. Child Dev. 2007, 78, 558-580. [CrossRef]

18. Hamre, B.; Hatfield, B.; Pianta, R.; Jamil, F. Evidence for general and domain-specific elements of teacher-child interactions: Associations with preschool children's development. Child Dev. 2014, 85, 1257-1274. [CrossRef]

19. Justice, L.M.; Mashburn, A.J.; Hamre, B.K.; Pianta, R.C. Quality of language and literacy instruction in preschool classrooms serving at-risk pupils. Early Child. Res. Q. 2008, 23, 51-68. [CrossRef]

20. LoCasale-Crouch, J.; Konold, T.; Pianta, R.; Howes, C.; Burchinal, M.; Bryant, D.; Clifford, R.; Early, D.; Barbarin, O. Observed classroom quality profiles in state-funded pre-kindergarten programs and associations with teacher, program, and classroom characteristics. Early Child. Res. Q. 2007, 22, 3-17. [CrossRef]

21. Pianta, R.C.; Barnett, W.S.; Burchinal, M.; Thornburg, K.R. The effects of preschool education: What we know, how public policy is or is not aligned with the evidence base, and what we need to know. Psychol. Sci. Public Interest 2009, 10, 49-88. [CrossRef]

22. Stuck, A.; Kammermeyer, G.; Roux, S. The reliability and structure of the Classroom Assessment Scoring System in German pre-schools. Eur. Early Child. Educ. Res. J. 2016, 24, 873-894. [CrossRef]

23. Weissberg, R.P.; Durlak, J.A.; Domitrovich, C.E.; Gullotta, T.P. Social and emotional learning: Past, present, and future. In Handbook of Social and Emotional Learning: Research and Practice; Durlak, J.A., Domitrovich, C.E., Weissberg, R.P., Gullotta, T.P., Eds.; The Guilford Press: New York, NY, USA, 2015.

24. Bierman, K.; Motamedi, M. Social and emotional learning programs for preschool children. In Handbook of Social and Emotional Learning: Research and Practice; Durlak, J.A., Domitrovich, C.E., Weissberg, R.P., Gullotta, T.P., Eds.; The Guilford Press: New York, NY, USA, 2015.

25. Collaborative for Academic, Social, and Emotional Learning. CASEL Guide: Effective Social and Emotional Learning Programs: Preschool and Elementary School Edition; CASEL: Chicago, IL, USA, 2013.

26. McClelland, M.M.; Tominey, S.L.; Schmitt, S.A.; Duncan, R. SEL interventions in early childhood. Future Child. 2017, 33-47. [CrossRef]

27. Sabey, C.V.; Charlton, C.T.; Pyle, D.; Lignugaris-Kraft, B.; Ross, S.W. A review of classwide or universal social, emotional, behavioral programs for students in kindergarten. Rev. Educ. Res. 2017, 87, 512-543. [CrossRef]

28. Schindler, H.S.; Kholoptseva, J.; Oh, S.S.; Yoshikawa, H.; Duncan, G.J.; Magnuson, K.A.; Shonkoff, J.P. Maximizing the potential of early childhood education to prevent externalizing behavior problems: A meta-analysis. J. Sch. Psychol. 2015, 53, 243-263. [CrossRef]

29. Blewitt, C.; Fuller-Tyszkiewicz, M.; Nolan, A.; Bergmeier, H.; Vicary, D.; Huang, T.; McCabe, P.; McKay, T.; Skouteris, H. Social and Emotional Learning Associated with Universal Curriculum-Based Interventions in Early Childhood Education and Care Centers: A Systematic Review and Meta-analysis. JAMA Netw. Open 2018, 1, e185727. [CrossRef]

30. Durlak, J.A. What everyone should know about implementation. In Handbook of Social and Emotional Learning: Research and Practice; Durlak, J.A., Domitrovich, C.E., Weissberg, R.P., Gullotta, T.P., Eds.; The Guilford Press: New York, NY, USA, 2015.

31. Conroy, M.A.; Sutherland, K.S.; Algina, J.J.; Wilson, R.E.; Martinez, J.R.; Whalon, K.J. Measuring Teacher Implementation of the "BEST in CLASS" Intervention Program and Corollary Child Outcomes. J. Emot. Behav. Disord. 2015, 23, 144-155. [CrossRef] 
32. Werner, C.D.; Linting, M.; Vermeer, H.J.; Van IJzendoorn, M.H. Do intervention programs in child care promote the quality of caregiver-child interactions? A meta-analysis of randomized controlled trials. Prev. Sci. 2016, 17, 259-273. [CrossRef]

33. Oberle, E.; Domitrovich, C.E.; Meyers, D.C.; Weissberg, R.P. Establishing systemic social and emotional learning approaches in schools: A framework for schoolwide implementation. Camb. J. Educ. 2016, 46, 277-297. [CrossRef]

34. Moher, D.; Liberati, A.; Tetzlaff, J.; Altman, D.G. Preferred reporting items for systematic reviews and meta-analyses: The PRISMA statement. Ann. Internal Med. 2009, 151, 264-269. [CrossRef]

35. Domitrovich, C.E.; Gest, S.D.; Gill, S.; Bierman, K.L.; Welsh, J.A.; Jones, D. Fostering High-Quality Teaching with an Enriched Curriculum and Professional Development Support: The Head Start REDI Program. Am. Educ. Res. J. 2009, 46, 567-597. [CrossRef]

36. Bierman, K.L.; DeRousie, R.M.S.; Heinrichs, B.; Domitrovich, C.E.; Greenberg, M.T.; Gill, S. Sustaining high-quality teaching and evidence-based curricula: Follow-up assessment of teachers in the REDI project. Early Educ. Dev. 2013, 24, 1194-1213. [CrossRef] [PubMed]

37. Thomas, B.; Ciliska, D.; Dobbins, M.; Micucci, S. A process for systematically reviewing the literature: Providing the research evidence for public health nursing interventions. Worldviews Evid. Based Nurs. 2004, 1, 176-184. [CrossRef] [PubMed]

38. Arda, T.B.; Ocak, S. Social Competence and Promoting Alternative Thinking Strategies-PATHS Preschool Curriculum. Educ. Sci. Theory Pract. 2012, 12, 2691-2698.

39. Barnett, W.S.; Jung, K.; Yarosz, D.J.; Thomas, J.; Hornbeck, A.; Stechuk, R.; Burns, S. Educational Effects of the Tools of the Mind Curriculum: A Randomized Trial. Early Child. Res. Q. 2008, 23, 299-313. [CrossRef]

40. Cappella, E.; O'Connor, E.E.; McCormick, M.P.; Turbeville, A.R.; Collins, A.J.; McClowry, S.G. Classwide Efficacy of INSIGHTS: Observed Teacher Practices and Student Behaviors in Kindergarten and First Grade. Elem. Sch. J. 2015, 116, 217-241. [CrossRef]

41. Fishbein, D.H.; Domitrovich, C.; Williams, J.; Gitukui, S.; Guthrie, C.; Shapiro, D.; Greenberg, M. Short-term intervention effects of the PATHS curriculum in young low-income children: Capitalizing on plasticity. J. Primary Prev. 2016, 37, 493-511. [CrossRef]

42. Jackman, M.M.; Nabors, L.A.; McPherson, C.L.; Quaid, J.D.; Singh, N.N. Feasibility, acceptability, and preliminary effectiveness of the openmind (om) program for pre-school children. J. Child Fam. Stud. 2019, 28, 2901-2921. [CrossRef]

43. Landry, S.H.; Zucker, T.A.; Taylor, H.B.; Swank, P.R.; Williams, J.M.; Assel, M.; Crawford, A.; Huang, W.; Clancy-Menchetti, J.; Lonigan, C.J.; et al. Enhancing early child care quality and learning for toddlers at risk: The responsive early childhood program. Dev. Psychol. 2014, 50, 526-541. [CrossRef]

44. Lonigan, C.J.; Phillips, B.M.; Clancy, J.L.; Landry, S.H.; Swank, P.R.; Assel, M.; Taylor, H.B.; Klein, A.; Starkey, P.; Domitrovich, C.E.; et al. Impacts of a comprehensive school readiness curriculum for preschool children at risk for educational difficulties. Child Dev. 2015, 86, 1773-1793. [CrossRef]

45. Upshur, C.C.; Heyman, M.; Wenz-Gross, M. Efficacy trial of the Second Step Early Learning (SSEL) curriculum: Preliminary outcomes. J. Appl. Dev. Psychol. 2017, 50, 15-25. [CrossRef]

46. Upshur, C.; Wenz-Gross, M.; Reed, G. A pilot study of a primary prevention curriculum to address preschool behavior problems. J. Primary Prev. 2013, 34, 309-327. [CrossRef] [PubMed]

47. Webster-Stratton, C.; Reid, M.J.; Stoolmiller, M. Preventing conduct problems and improving school readiness: Evaluation of the Incredible Years teacher and child training programs in high-risk schools. J. Child Psychol. Psychiatry 2008, 49, 471-488. [CrossRef] [PubMed]

48. Gunter, L.; Caldarella, P.; Korth, B.B.; Young, K.R. Promoting social and emotional learning in preschool students: A study of Strong Start Pre-K. Early Child. Educ. J. 2012, 40, 151-159. [CrossRef]

49. Pickens, J. Socio-Emotional Programme Promotes Positive Behaviour in Preschoolers. Child Care Pract. 2009, 15, 261-278. [CrossRef]

50. Seyhan, G.; Ocak Karabay, S.; Arda Tuncdemir, T.B.; Greenberg, M.T.; Domitrovich, C. The effects of promoting alternative thinking strategies preschool program on teacher-children relationships and children's social competence in turkey. Int. J. Psychol. 2017, 54, 61-69. [CrossRef]

51. Vestal, A.; Jones, N.A. Peace Building and Conflict Resolution in Preschool Children. J. Res. Child. Educ. 2004, 19, 131-142. [CrossRef] 
52. Denham, S.A.; Burton, R. Social and Emotional Prevention and Intervention Programming for Preschoolers; Kluwer-Plenum: New York, NY, USA, 2003.

53. Barnett, W.S. Effectiveness of early educational intervention. Science 2011, 333, 975-978. [CrossRef]

54. Oberklaid, F.; Baird, G.; Blair, M.; Melhuish, E.; Hall, D. Children's health and development: Approaches to early identification and intervention. Arch. Dis. Child. 2013, 98, 1008-1011. [CrossRef]

55. Yoshikawa, H.; Weiland, C.; Brooks-Gunn, J.; Burchinal, M.R.; Espinosa, L.M.; Gormley, W.T.; Ludwig, J.; Magnuson, K.A.; Phillips, D.; Zaslow, M.J. Investing in Our Future: The Evidence Base on Preschool Education; Society for Research in Child Development and Foundation for Child Development: Ann Arbor, MI, USA, 2013.

56. Buettner, C.K.; Jeon, L.; Hur, E.; Garcia, R.E. Teachers' Social-Emotional Capacity: Factors Associated with Teachers' Responsiveness and Professional Commitment. Early Educ. Dev. 2016, 27, 1018-1039. [CrossRef]

57. Mashburn, A.J.; Pianta, R.C.; Hamre, B.K.; Downer, J.T.; Barbarin, O.A.; Bryant, D.; Burchinal, M.; Early, D.M.; Howes, C. Measures of Classroom Quality in Prekindergarten and Children's Development of Academic, Language, and Social Skills. Child Dev. 2008, 79, 732-749. [CrossRef]

58. Curby, T.W.; LoCasale-Crouch, J.; Konold, T.R.; Pianta, R.C.; Howes, C.; Burchinal, M.; Bryant, D.; Clifford, R.; Early, D.; Barbarin, O. The Relations of Observed Pre-K Classroom Quality Profiles to Children's Achievement and Social Competence. Early Educ. Dev. 2009, 20, 346-372. [CrossRef]

59. Brock, L.L.; Curby, T.W. Emotional support consistency and teacher-child relationships forecast social competence and problem behaviors in prekindergarten and kindergarten. Early Educ. Dev. 2014, 25, 661-680. [CrossRef]

60. Hamre, B.K. Teachers' daily interactions with children: An essential ingredient in effective early childhood programs. Child Dev. Perspect. 2014, 8, 223-230. [CrossRef]

61. Ainsworth, M.D.S.; Blehar, M.C.; Waters, E.; Wall, S.N. Patterns of Attachment: A Psychological Study of the Strange Situation; Psychology Press: New York, NY, USA, 2015.

62. Rimm-Kaufman, S.E.; Curby, T.W.; Grimm, K.J.; Nathanson, L.; Brock, L.L. The contribution of children's self-regulation and classroom quality to children's adaptive behaviors in the kindergarten classroom. Dev. Psychol. 2009, 45, 958. [CrossRef] [PubMed]

63. Sheridan, S.M.; Edwards, C.P.; Marvin, C.A.; Knoche, L.L. Professional development in early childhood programs: Process issues and research needs. Early Educ. Dev. 2009, 20, 377-401. [CrossRef]

64. Fukkink, R.G.; Lont, A. Does training matter? A meta-analysis and review of caregiver training studies. Early Child. Res. Q. 2007, 22, 294-311. [CrossRef]

65. Whiting, P.; Savović, J.; Higgins, J.P.T.; Caldwell, D.M.; Reeves, B.C.; Shea, B.; Davies, P.; Kleijnen, J.; Churchill, R. ROBIS: A new tool to assess risk of bias in systematic reviews was developed. J. Clin. Epidemiol. 2016, 69, 225-234. [CrossRef]

(C) 2020 by the authors. Licensee MDPI, Basel, Switzerland. This article is an open access article distributed under the terms and conditions of the Creative Commons Attribution (CC BY) license (http://creativecommons.org/licenses/by/4.0/). 